\title{
Light Capacitances in Silicon and Perovskite Solar Cells
}

\author{
Osbel Almora ${ }^{\mathrm{a}, \mathrm{b}, \mathrm{c}}$ and Germà Garcia-Belmonte ${ }^{\mathrm{a}, *}$ \\ a Institute of Advanced Materials (INAM), Universitat Jaume I, 12006 Castelló, Spain \\ ${ }^{\mathrm{b}}$ Institute of Materials for Electronics and Energy Technology (i-MEET), Friedrich-Alexander- \\ Universität Erlangen-Nürnberg, 91058 Erlangen, Germany \\ ${ }^{c}$ Erlangen Graduate School in Advanced Optical Technologies (SAOT), Friedrich-Alexander- \\ Universität Erlangen-Nürnberg, 91052 Erlangen, Germany
}

\begin{abstract}
The framework on which the physics of silicon solar cells (SiSCs) is based robustly predicts dependences of capacitance on light intensity and voltage, even when most recent innovations are considered as the incorporation of transition metal oxide/Si heterojunctions. However, perovskite solar cells (PSCs) challenge most of the established paradigms, claiming for rethinking of known theories and devising novel models. Here we tackle this scenario by probing and comparing light-induced capacitance responses yielded by these two major exponents in the field of photovoltaic research. SiSCs light capacitances can be easily interpreted in the framework of the so-called chemical capacitance. Current approaches addressing the intriguing low-frequency capacitive features of PSCs are outlined and compared. Here, apparent similarities and differences between both photovoltaic technologies are highlighted, concerning the observation of light capacitances of chemical origin. It is concluded that, contrary to that occurring in SiSCs, bulk electronic chemical capacitances are not straightforwardly observed in PSCs. As capacitive features exhibited by PSCs are believed to be critically connected to performance degradation and device instability, future research and
\end{abstract}


explanation directions are proposed here for advancing in the understanding of this challenging photovoltaic technology.

Keywords: Capacitance, light, solar cells, silicon, perovskites, dielectric relaxation.

Corresponding author: garciag@uji.es 


\section{Introduction}

In recent years, perovskite solar cells (PSCs), incorporating hybrid metal halide perovskite compounds as methylammonium lead iodide $\left(\mathrm{MAPbI}_{3}\right)$ (Kim et al., 2012; Lee et al., 2012; Yang et al., 2015) and other combinations (Saliba et al., 2016), have achieved power conversion efficiencies above $24 \%$ (Green et al., 2019), comparable to those exhibited by silicon solar cells (SiSCs). The impressive optoelectronic performance of PSCs stems from the reported high absorption coefficient, long charge diffusion length and high carrier mobility, reduced recombination rate, low exciton binding energy, and tunable direct band gap (Green et al., 2014; Oga et al., 2014; Park, 2015; Wehrenfennig et al., 2014; Zhao and Zhu, 2016). Despite their superior optoelectronic properties, there remains a set of unresolved issues that block commercialization of PSCs, mainly related to their chemical stability and photovoltaic parameter degradation/variation. Among other operating alterations, there is extensive literature concerning the hysteretic effects on the current density-voltage $(J-V)$ characteristics (Jeon et al., 2014; Snaith et al., 2014; Unger et al., 2014). Hysteresis has a detrimental influence on the photovoltaic operation, device reliability and stability so as to advance in its alleviation. This technological drawback was suggested to be connected to their uncommon capacitive and photo-capacitive response (Almora et al., 2016).

Light capacitance in PSCs was reported to exhibit an intriguing behavior in the lowfrequency part of the capacitive spectrum (Juarez-Perez et al., 2014). A huge increment of capacitance is commonly encountered at $0.1-10 \mathrm{~Hz}$, which results proportional to the light intensity, both in short- and open-circuit conditions (Juarez-Perez et al., 2014; Zarazua et al., 2016a). Values as high as $0.1 \mathrm{~F} \mathrm{~cm}^{-2}$ can be readily achieved at standard 1 sun illumination, a feature hardly observed in other kind of solar cell technologies. It was 
early noted that even in the dark, PSCs shows relatively large capacitances $(\sim 50 \mu \mathrm{F} \mathrm{cm}-2)$ at low frequencies as compared with geometrical capacitance, irrespective of the perovskite bulk thickness (Almora et al., 2015). This fact indicated that interfaces between the absorbing semiconductor and contacting layers would be behind these uncommon capacitive experimental trends (Bergmann et al., 2016; Carrillo et al., 2016; Kim et al., 2017). Simultaneously, it was reported a hysteretic effect of the $J$ - $V$ curve under operation. It has been ascribed to several mechanisms: intrinsic ferroelectricity of the perovskite compounds (Wei et al., 2014), delayed trap filling upon illumination (Shao et al., 2014), ionic charge accumulation at the outer interfaces (Almora et al., 2019b; Li et al., 2016; Meloni et al., 2016). The connection between most significant hysteresis trends and the capacitive features has been firmly established (Chen et al., 2015). It is also known that photovoltaic perovskites possess a non-negligible ionic conductivity (Azpiroz et al., 2015; Meloni et al., 2016). This fact has suggested the participation of mobile ionic species in the explanation of either the achieved level (Kim et al., 2018) or the slow kinetics (Bag et al., 2015) of the observed hysteresis and photo-capacitive effect. Also the formation of an electronic space-charge accumulation zone at the interfaces between the perovskite and contacting layers has been proposed (Zarazua et al., 2016a). In any case, the capacitive issue does not have a widely accepted and shared explanation. But it deserves further attention as light capacitance exhibited by PSCs is connected to performance degradation and device instability. Therefore, a deep analysis of the capacitive and photocapacitive response in PSCs is needed and here it is compared with the capacitance behavior of a well-known technology such as the silicon solar cell. 
Standard Physics establishes two types of well-known capacitive mechanisms. On the one hand, semiconductors possess a given polarizability, which establishes the amount of surface charge per unit area that can be stored by effect of the applied voltage. This charge is characterized by the dielectric geometrical capacitance $\left(C_{g}\right)$, and the depletion layer capacitance $C_{d l}$ if present (Almora et al., 2019a), and is often easily accessed by basic impedance spectroscopy techniques (IS) (Lopez-Varo et al., 2018). In addition, for some semiconductors it is also possible to storage charge and energy directly in the bulk region between the metallic plates/electrodes. This charge accumulation can be characterized by the concept of chemical capacitance $\left(C_{\mu}\right)$ (Bisquert, 2003). It establishes the effect of Fermi level displacements on the occupancy change in the density-of-states (DOS) of electronic charge carriers. For a small perturbation regime, and assuming that the output voltage easily connects to the internal Fermi level, the response accounting for charge storage is essentially capacitive in nature (Bisquert, 2014). This is particularly observed when effective electrical fields are shielded, either because a large density of majorities or compensating mobile charges.

Here we survey on the established theories about common capacitive features under illumination observed in current photovoltaic technologies. Especially important is the discussion on the conditions of observation of the chemical capacitance. As subsequently explained, the capacitive response of silicon solar cells (SiSCs) is easily connected to the charge carrier DOS occupancy and, consequently, to that predicted by the chemical capacitance framework. After that, we comment on the new phenomena and state of the art research regarding the trending field of perovskite solar cells (PSCs), in terms of its unique capacitive response. We outline here their distinctive features with respect to the 
well-established SiSC technology and point out apparent similarities and differences concerning the observation of light capacitances of chemical origin.

\section{Chemical capacitance measuring conditions}

The first necessary condition to be fulfilled by a given semiconductor material in order to assure the observation of chemical capacitance features relates to the competition between dielectric relaxation time and carrier recombination time. Long relaxation times, of the order of or exceeding recombination times, entails carriers slowly achieve steady-state conditions. As chemical capacitance is measured by small perturbation of a given steady state, it is necessary that the carriers in the semiconductor bulk dielectrically relax before measurement. This also relates the presence or absence of extended quasi-neutral regions within the active layer bulk. The dielectric relaxation time can be expressed in terms of the material permittivity $\mathcal{E} \varepsilon_{0}$ (being $\mathcal{E}$ the dielectric constant, and $\varepsilon_{0}$ the vacuum permittivity), and its conductivity $\sigma$ as

$\tau_{\text {die }}=\frac{\varepsilon \varepsilon_{0}}{\sigma}$

The occurrence of quasi-neutral regions relies on the property that carrier lifetime (recombination time, $\tau_{r e c}$ ) is much larger than the dielectric relaxation time $\tau_{d i e}$. If $\tau_{\text {rec }}>\tau_{\text {die }}$ mobile carriers can exist long enough to neutralize net charge and suppress space-charge regions. Depending on whether $\tau_{\mathrm{rec}}>\tau_{\mathrm{die}}$ (lifetime semiconductor regime), or $\tau_{\mathrm{rec}}<\tau_{\mathrm{die}}$ (relaxation semiconductor regime) the physics governing the device operation changes drastically (Fonash, 2010). In the relaxation semiconductor regime, electro-neutrality is not a justifiable assumption. Therefore, space charge of dielectric relaxation-dependent decay is allowed to occur (van 
Roosbroeck and Casey, 1972). Low-conductivity amorphous $p-i-n$ SiSCs develop photogenerated hole space charge regions near the $p$ contact that concentrate the voltage drop (Schiff, 2003). In the extreme case currents should be space charge limited as occurring in organic lightemitting diodes based on low-mobility polymers or molecules (Bozano et al., 1999).

A device will be then functioning in lifetime or relaxation regime depending on the materials properties and operating conditions. For doped enough semiconductors, $\tau_{\text {die }}<<\tau_{\text {rec }}$ because enhanced conductivity, so as to shrink the depletion zone built in the vicinity of the contacts at operation voltages close to the maximum power point. This leaves extended neutral regions within the semiconductor bulk in which transport of minority carrier should be controlled by diffusion (Burgelman and Grasso, 2004) while the high conductivity of majority carriers assures rapid shielding. Fig. 1 illustrates these operating regimes in semiconductor devices. Also in terms of characteristic lengths, the relaxation semiconductor regime $\left(\tau_{\text {die }}>\tau_{\text {rec }}\right)$ can in turn be expressed as $\lambda_{D}>L_{\mu}$, which indicates longer Debye lengths $\lambda_{D}=\sqrt{D_{\mu} \tau_{\text {die }}}$ than diffusion lengths $L_{\mu}$, being $D_{\mu}$ the carrier diffusion coefficient. 


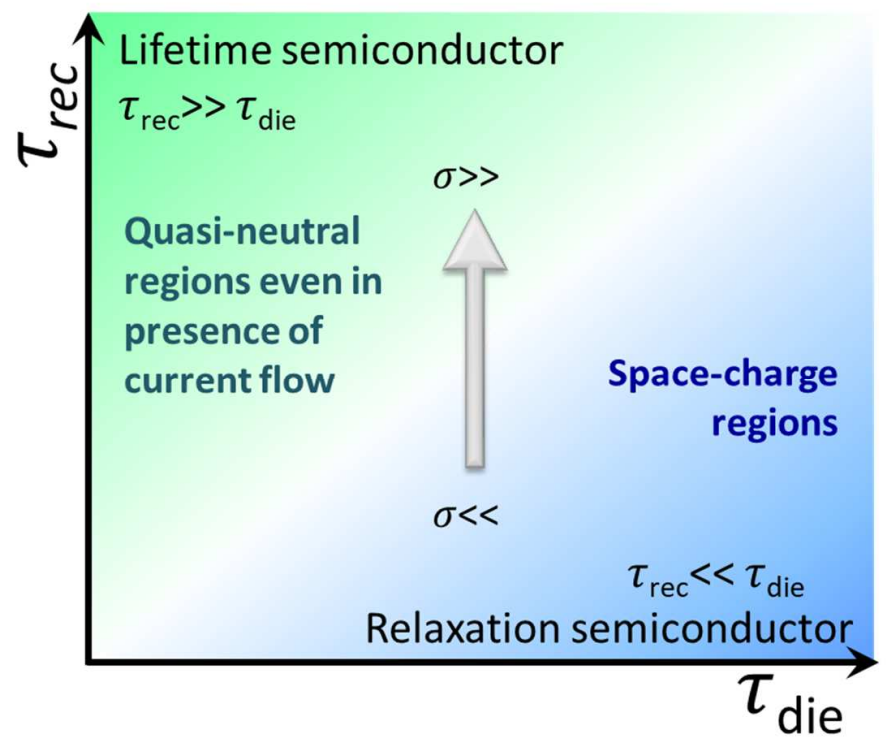

Fig. 1. Scheme on semiconductor operating regimes regarding the relationship between characteristic semiconductor times.

Existence of quasi-neutral regions inside the semiconductor bulk is then a necessary condition to unambiguously measure capacitances of chemical origin. The archetypical photovoltaic device showing chemical capacitances is silicon $p$ - $n$ solar cell. Wide absorbing electro-neutral regions of width $L$ storage carriers under illumination and forward voltages. This effect is maximized in open-circuit when no $d c$ current is allowed to flow. Light- or voltage-induced occupancy changes of conduction band electrons and valence band holes imply the splitting of quasi-Fermi levels (electron and hole) that, in the simplest case of large enough conductivities, is related to the output voltage as

$q V=E_{F n}-E_{F p}$ 
being $q$ the elementary charge. The general expressions for electron $n$ and hole $p$ concentrations derive from Boltzmann statistics, electro-neutrality conditions and the massaction law, is written as (Brendel, 2005)

$$
\begin{aligned}
& n=\frac{N_{D}-N_{A}}{2}+\sqrt{\left(\frac{N_{D}-N_{A}}{2}\right)^{2}+n_{i}^{2} \exp \left[\frac{E_{F n}-E_{F p}}{k_{B} T}\right]} \\
& p=\frac{N_{A}-N_{D}}{2}+\sqrt{\left(\frac{N_{A}-N_{D}}{2}\right)^{2}+n_{i}^{2} \exp \left[\frac{E_{F n}-E_{F p}}{k_{B} T}\right]}
\end{aligned}
$$

Here, $N_{D}$ and $N_{A}$ accounts for the ionized donor and acceptor impurity concentrations, $n_{i}$ is the intrinsic carrier density, and $k_{\mathrm{B}} T$ represents the thermal energy. Excess carrier concentration produced by light irradiation or bias voltage can be accessed by exploring chemical capacitive effects. The chemical capacitance (Bisquert, 2014), also known as diffusion capacitance (Sze and $\mathrm{Ng}, 2007$ ), informs on the occupancy of conduction band bulk electrons (Bisquert et al., 2004) as

$$
C_{\mu n}=q^{2} L \frac{d n}{d E_{F n}}
$$

where the capacitance is given per unit area. Since at forward bias or under usual illumination levels the device can work in high-injection conditions, the occupancy change of valence band bulk holes gives rise to an additional capacitance per unit area as

$$
C_{\mu p}=-q^{2} L \frac{d p}{d E_{F p}}
$$

Accordingly, from equation (4) and taking into account equation (3) (Brendel, 2005), which relates the splitting of the quasi-Fermi levels and the carrier concentrations at a given 
temperature, one can take directly the partial derivatives and the capacitance per unit area can be expressed as (Mora-Seró et al., 2008)

$$
C_{\mu n}=\frac{q^{2} L_{n}}{2 k_{B} T} \frac{n_{i}^{2} \exp \left[\frac{E_{F n}-E_{F p}}{k_{B} T}\right]}{\sqrt{\left(\frac{N_{D}-N_{A}}{2}\right)^{2}+n_{i}^{2} \exp \left[\frac{E_{F n}-E_{F p}}{k_{B} T}\right]}}
$$

And similarly, in the case of bulk holes

$$
C_{\mu p}=\frac{q^{2} L_{p}}{2 k_{B} T} \frac{n_{i}^{2} \exp \left[\frac{E_{F n}-E_{F p}}{k_{B} T}\right]}{\sqrt{\left(\frac{N_{A}-N_{D}}{2}\right)^{2}+n_{i}^{2} \exp \left[\frac{E_{F n}-E_{F p}}{k_{B} T}\right]}}
$$

Here lengths $L_{n}$ and $L_{p}$ account as corrections on the effective quasi-neutral region widths, in relation to the bulk thickness $L$, due to asymmetric partial derivatives effects or deviations from equation 3.

It is important to note that equation (5) predicts two limiting regimes depending on the relation between excess carriers and doping level. For a $p$-type semiconductor and minority electron concentration $n<<N_{A}$, electron chemical capacitance obeys the exponential relation $C_{\mu n} \propto \exp \left(V / k_{\mathrm{B}} T\right)$. On the contrary, when $n>>N_{A}$ the exponential law with voltage should behave as $C_{\mu n} \propto \exp \left(V / 2 k_{\mathrm{B}} T\right)$. Therefore, a transition between two regimes in the exponential slope from $1 / k_{\mathrm{B}} T$ to $1 / 2 k_{\mathrm{B}} T$ is expected, if logarithm scaled capacitance is plotted as a function of the open-circuit voltage (Fermi level splitting) of equation (2). It is important to stress that equation (5) will be readily of application in open-circuit conditions, since this maximizes the charge storage in the absorbing material by suppressing $d c$ current flow and transport gradients. 
We also note that the introduced expressions assume large carrier mobility, homogeneous carrier density profiles and perfectly selective contacts (Mora-Sero et al., 2009).

The experimental observation of the chemical capacitance is also restricted by resistive processes occurring in series with respect to it. As equation (5) refers to the total frequency-independent chemical capacitances of the device, the practical measurement of this capacitances by IS depends on the coupled resistance $(R)$, which may shift the spectra toward lower or higher frequencies, depending on the measuring response frequency $f=\tau^{-1}=(R C)^{-1}$. In other words, the larger the resistivity, the lower are the frequencies at which chemical capacitances could be measured, as illustrated in Fig. 2.

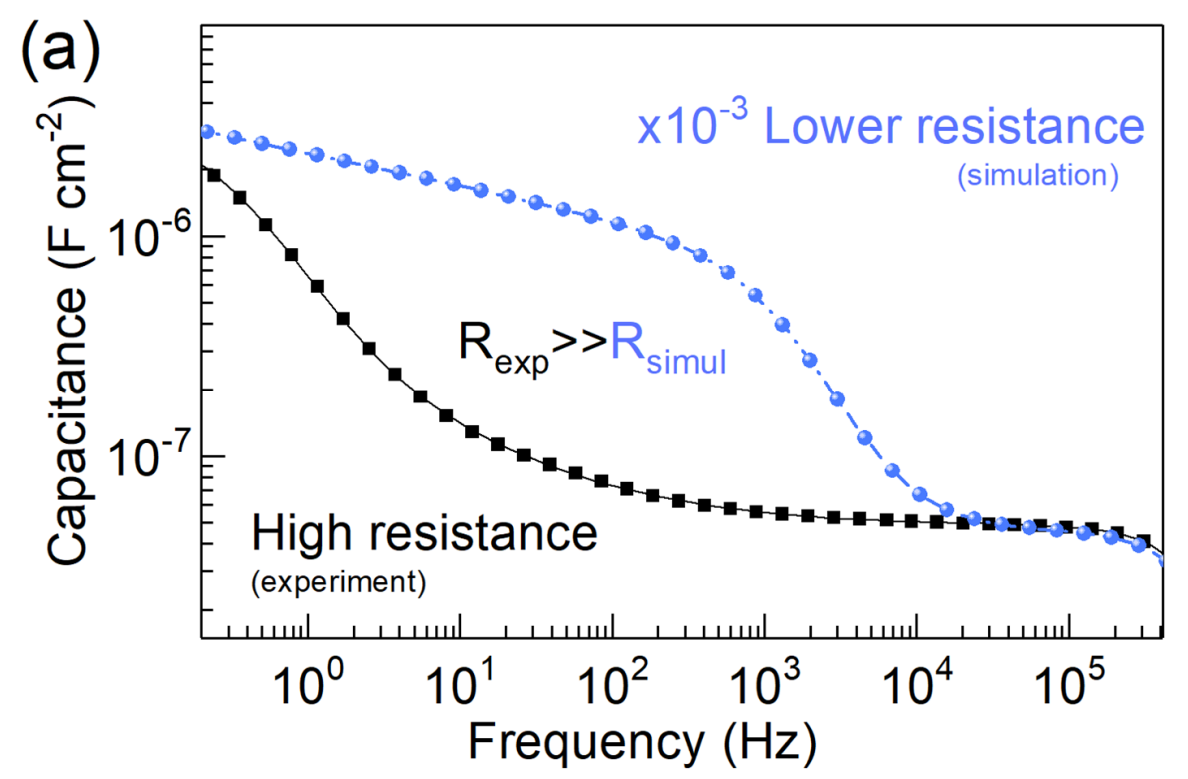

Fig. 2. Capacitance spectra of a perovskite solar cell and simulated frequency shifted spectrum, showing the masking effect of resistances when measuring IS. Modified with permission from ref. (Almora et al., 2018a), Copyright (2018) American Chemical Society. 


\section{Chemical capacitance in Si solar cells}

For SiSCs, the relationships of equation (5) were reported in the case of heterojunction devices incorporating either thin hydrogenated amorphous silicon (a-Si:H) films deposited on crystalline silicon (c-Si) wafers (Mora-Seró et al., 2008), or transition metal oxides as selective contacts (Almora et al., 2017a). As an example, we show in Fig. 3 electron and hole chemical capacitances exhibited by a heterojunction SiSC along with the theoretical fitting using equation (5).

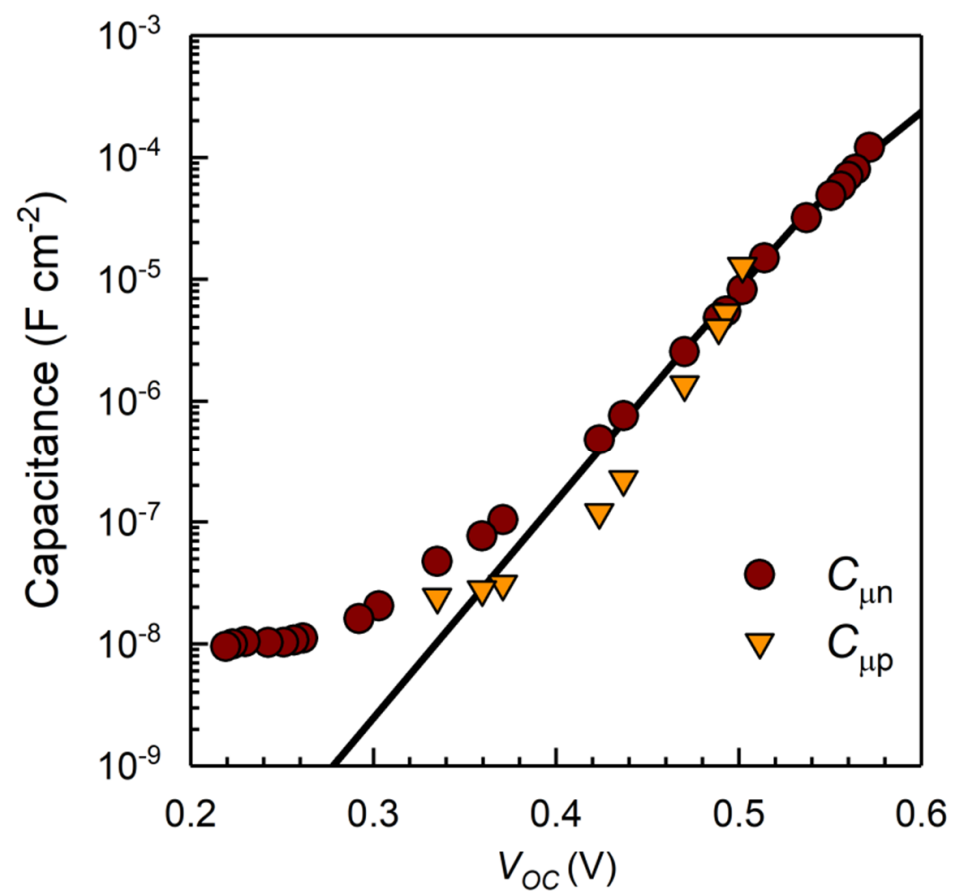

Fig. 3. Electron and hole chemical capacitances, $C_{n}$ and $C_{p}$, as a function of $V_{\mathrm{oc}}$. Solid line corresponds to equation (5) by assuming intrinsic carrier concentration $n_{i}=10^{10} \mathrm{~cm}^{-3}$, wafer $p$-doping $N_{A}=2 \times 10^{15} \mathrm{~cm}^{-3}$, wafer thickness $L=300 \mu \mathrm{m}$, and room temperature $T=290 \mathrm{~K}$. Adapted with permission from ref. (MoraSeró et al., 2008), Copyright (2008) Elsevier. 
Well-separated electron and hole chemical capacitances are not always observable, and one of the chemical capacitances dominate (Almora et al., 2017a; Mora-Sero et al., 2009). Only when the chemical capacitive effect is measured in a sufficiently wide frequency window, the unambiguous extraction of the capacitance parameters is feasible. It is also noted that the chemical capacitance response is masked by the capacitive contribution of the contact, as observed in Fig. 3 for low voltages $V<0.3 \mathrm{~V}$. The space-charge depletion zone in the vicinity of the contact produces an additional capacitance in excess of the chemical capacitance that is therefore invisible.

In other cases is even possible observing the regime change in the exponential slope from $1 / k_{\mathrm{B}} T$ to $1 / 2 k_{\mathrm{B}} T$ (Almora et al., 2017a), as shown in Fig. 4a for a $\mathrm{Si} / \mathrm{V}_{2} \mathrm{O}_{5}$ heterojunction device. Furthermore, Fig. $4 \mathrm{~b}$ presents the spectra corresponding to a $\mathrm{Si} / \mathrm{MoO}_{3}$ heterojunction solar cell in the analogue situation to Fig. $4 \mathrm{a}$. There, the characteristic capacitance plateaus around $1 \mathrm{kHz}$ in the capacitance spectra exhibit growing values by more than two orders of magnitude under light irradiation, as expected from equation (5) in the corresponding $V_{o c}$ range. The effect of the diminishing series resistance is also evidenced as a reduction in capacitance at higher frequencies.

Interestingly, another consequence of equation (5) is that in short-circuit condition ( $\left.V=\left(E_{F n}-E_{F p}\right) / q=0\right)$ no increment in the capacitance is expected. This is nicely confirmed in the experiment, as shown in Fig. 4c for the capacitance of both a Si/ $\mathrm{V}_{2} \mathrm{O}_{5}$ device and a commercial Si homojunction solar cell at different illumination intensities. Only a slight increase of a few $\mathrm{nF}$ $\mathrm{cm}^{-2}$ appears, a typical the geometrical capacitance thermal effect. 
All these evidences obviously agree with the recombination character of doped silicon as used in solar cell technologies. A simple calculation considering equation (1), and doping densities approaching $10^{15} \mathrm{~cm}^{-3}$ for monocrystalline $\mathrm{Si}$, yields relaxation times approximately equal to $\tau_{\text {die }} \approx 7 \mathrm{~ns}$, which is orders of magnitude shorter than typical recombination times in the range of $\mu \mathrm{s}$. 

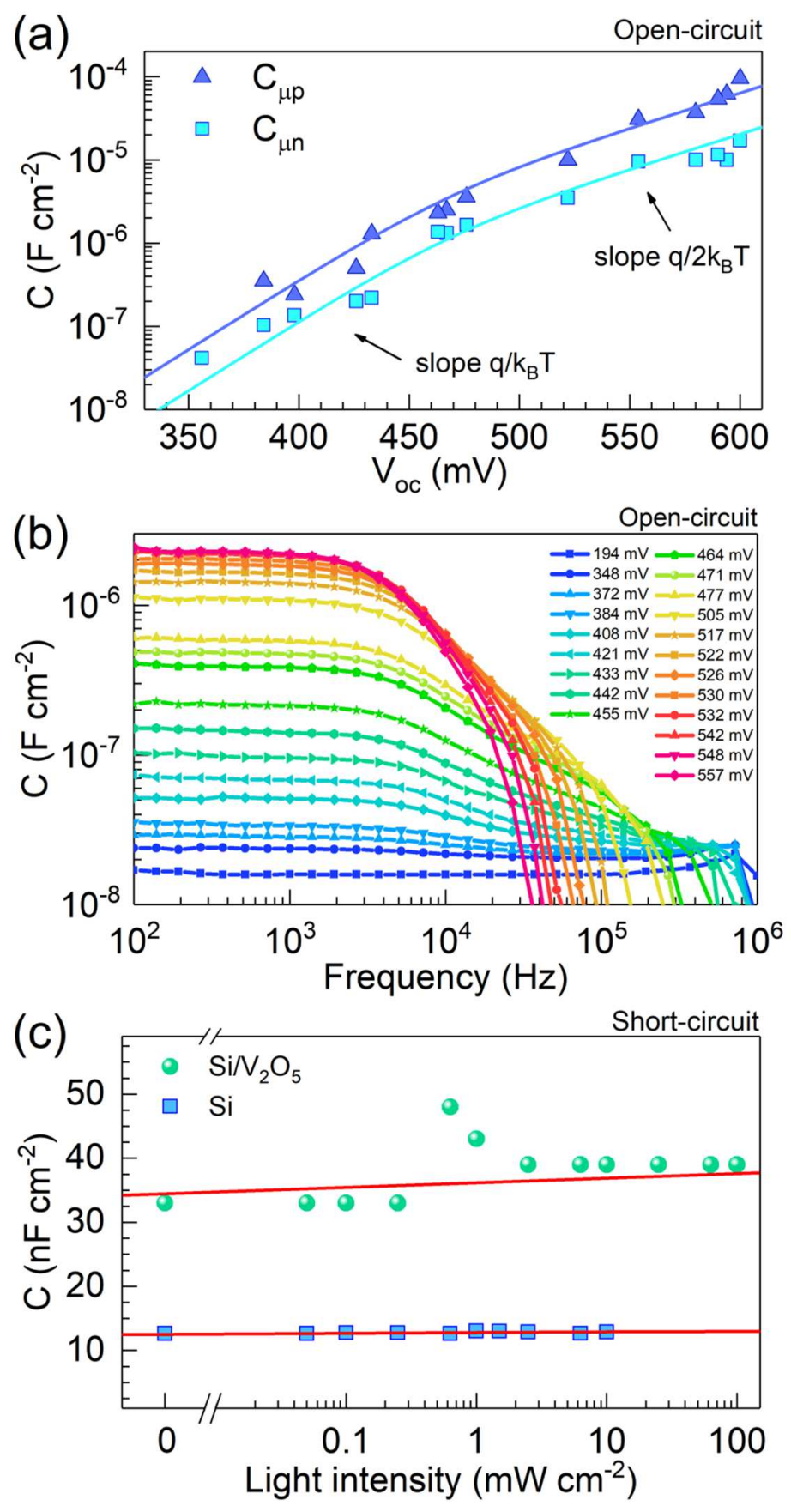

Fig. 4. (a) Electron and hole chemical capacitances as a function of $V_{\mathrm{oc}}$ of a heterojunction SiSC with textured $\mathrm{V}_{2} \mathrm{O}_{5}$ selective contact. Solid line corresponds to equation (5) by assuming intrinsic carrier concentration $n_{i}=1.5 \times 10^{10} \mathrm{~cm}^{-3}$, wafer $n$-doping $N_{D}=2.5 \times 10^{14} \mathrm{~cm}^{-3}, \quad L_{p}=125 \mu \mathrm{m}, \quad L_{n}=40 \mu \mathrm{m}$ and room temperature $T=300 \mathrm{~K}$. (b) Capacitance spectra as a function of $V_{o c}$ of a silicon heterojunction solar cell with $\mathrm{MoO}_{3}$ selective contact. (c) Capacitance (nearly constant) as a function of illumination intensities in 
short-circuit for the cell in (a) and a commercial mono crystalline SiSC. Adapted with permission from ref. (Almora et al., 2017a), Copyright (2017) Elsevier.

\section{The unexpected capacitive phenomena of perovskite solar cells}

Perovskite solar cells most commonly exhibit two capacitive responses as a function of the measuring frequency (Fig. 5a). A transition occurs between geometrical capacitances dominating at high frequencies to a light- and voltage-dependent low-frequency increment. As already noted, capacitance increments following an exponential law with voltage have been typically understood in terms of chemical capacitances. Therefore, it is in principle appealing trying to correlate the light-induced increase of the low-frequency $(0.1-1 \mathrm{~Hz})$ capacitance usually observed for perovskite-based solar cells (Fig. 5a) with the occurrence of chemical capacitance features. Note that the exponential increase of capacitance does not take place above $100 \mathrm{~Hz}$ as it is the case of SiSCs (see the example of Fig. 4b). Some experimental data show a slightly enhanced capacitance in PSCs for the intermediate frequency plateau of the capacitance spectra. This is also illustrated in Fig. 5a in which a capacitive plateau appears at $10^{3}-10^{4} \mathrm{~Hz}$ that increases with light. However, the increment hardly attains one order of magnitude, certainly small for the exponential dependences of equation (5), and several measurement factors could be considered as contributing to it (Almora et al., 2018a).

The light-induced exponentially-growing low-frequency capacitance $\left(C_{L f}\right)$ at open-circuit is illustrated in Fig. 5b for a set of PSCs comprising 3D perovskite layers based on $\mathrm{CH}_{3} \mathrm{NH}_{3} \mathrm{PbI}_{3}$ and a variety of interlayers (2D perovskite thin capping). By examining Fig. 5b, it also noted that the ground capacitance $C_{L f 0}$ from 0 to $0.4 \mathrm{~V}$ is not $C_{g}$, but a different surface capacitance 
possibly related with electrode polarization (irrespective of the bulk thickness), as introduced in previous papers (Almora et al., 2015).

At a first glance, the slope equaling $1 / 2 k_{\mathrm{B}} T$ in Fig. $5 \mathrm{~b}$ does not result conflictive with equation (5). Certainly, for low doped semiconductors and in high-injection (high enough illumination) it is derived that $N_{\mathrm{A}, \mathrm{D}}<<n_{i} \exp \left(E_{F n}-E_{F p} / k_{\mathrm{B}} T\right)$, and therefore equation (5) approximates to

$$
C_{\mu}=\frac{q^{2} L}{2 k_{B} T} n_{i} \exp \left[\frac{E_{F n}-E_{F p}}{2 k_{B} T}\right]
$$

However, it is certainly not the case for perovskite solar cells, as next explained. 

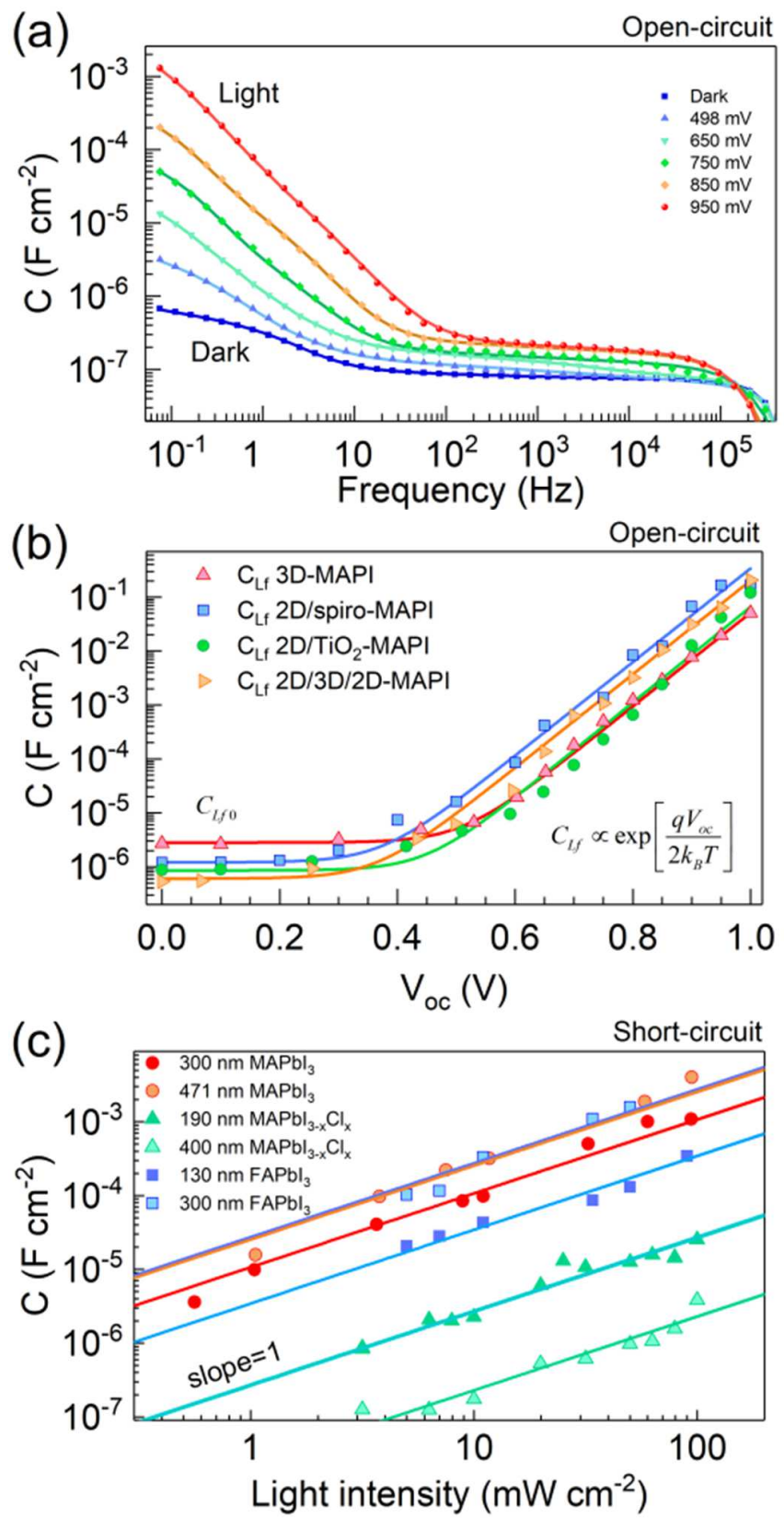

Fig. 5. (a) Capacitance spectra as a function of $V_{\mathrm{oc}}$ for a $\mathrm{CH}_{3} \mathrm{NH}_{3} \mathrm{PbI}_{3}$-based perovskite solar cell. (b) Low-Frequency capacitance for several devices based on $\mathrm{CH}_{3} \mathrm{NH}_{3} \mathrm{PbI}_{3}$ and a variety of interlayers (2D perovskite thin capping) also as a function of $V_{\mathrm{oc}}$. Adapted with permission from ref. (Almora et al., 2018b), Copyright (2018) Elsevier. (c) Capacitance directly proportional to illumination intensity in short-circuit condition for several perovskite solar cells. Adapted with permission from ref. (Zarazua et al., 2016b), Copyright (2016) American Chemical Society. 
Generally in $\mathrm{CH}_{3} \mathrm{NH}_{3} \mathrm{PbI}_{3}$-based PSCs, the low-frequency capacitance has been found (Almora et al., 2018b; Contreras-Bernal et al., 2017; Zarazua et al., 2016b; Zarazua et al., 2017) to follow $C \propto \exp \left(V / 2 k_{\mathrm{B}} T\right)$, with exactly the exponential slope $1 / 2 k_{\mathrm{B}} T$, as shown in Fig. 5b. The capacitance usually attains values as high as $C \approx 100 \mathrm{mF} \cdot \mathrm{cm}^{-2}$ for illumination intensity approaching 1 sun with $V_{o c} \approx 1.0 \mathrm{~V}$. For a typical perovskite absorbing layer of $L=300 \mathrm{~nm}$, one can infer from equation (6) that $n_{i} \approx 10^{12} \mathrm{~cm}^{-3}$. But this large intrinsic carrier density is in conflict with the value of $n_{i} \approx 10^{5}-10^{6} \mathrm{~cm}^{-3}$ inferred for the parabolic band approximation $n_{i}=\sqrt{N_{\mathrm{C}} N_{\mathrm{V}}} \exp \left(-E_{g} / 2 k_{\mathrm{B}} T\right)$, with effective conduction $N_{\mathrm{C}}$ and valence $N_{\mathrm{v}}$ band carrier densities of order $10^{18} \mathrm{~cm}^{-3}$ (Giorgi et al., 2013; Menéndez-Proupin et al., 2014) and band-gap $E_{g} \approx 1.6 \mathrm{eV}$ (Bisquert et al., 2016; LopezVaro et al., 2018). Several works have confirmed the low effective DOS of the conduction band in perovskites by Burstein-Moss shift (Manser and Kamat, 2014). It is then inferred that the low effective DOS values make it difficult to assign the low-frequency capacitive mechanism to the storage of excess photo-generated carriers occurring in the perovskite semiconductor bulk. In fact, by using equation (6) and $n_{i} \approx 10^{5}-10^{6} \mathrm{~cm}^{-3}$, one would expect chemical capacitances of only of the order of $C_{\mu} \approx 10^{-6} \mathrm{~F} \mathrm{~cm}^{-2}$ for $V_{o c} \approx 1.0 \mathrm{~V}$ (Bisquert et al., 2016). A value certainly small in comparison to the measured low-frequency capacitances, and slightly larger than that attained by the geometrical capacitance that depends on the dielectric properties of the perovskite as $C_{g}=\varepsilon \varepsilon_{0} / L$. A second reason reinforcing this conclusion is the very low frequencies $(0.1-1 \mathrm{~Hz})$ of observation, hardly connected to a pure electronic bulk process (Almora et al., 2019a).

In addition, equation (6) also fails to predict the linear increase of the low-frequency capacitance (in short-circuit conditions) with the light intensity or photocurrent, as shown in Fig. 5c for several PSCs including different material absorbers and absorber thicknesses. This behavior is 
drastically different to that occurring in SiSCs (see Fig. 5c) and is considered a distinctive feature of PSCs.

\section{Ionic-electronic coupling}

Provided the physical inconsistency of attributing a bulk-related chemical capacitance origin to the low-frequency capacitive response, we can infer that $(i)$ chemical capacitance are not observed at all in PSCs or (ii) equation (5) should be generalized in order to include extra influences on the effective DOS occupancies and charge distributions, e.g. mobile ions, even with a change of underlying mechanism. For instance, the low-frequency light capacitance features have been recently connected to the formation of electronic accumulation zones near the outer contacts (Zarazua et al., 2016a). That model restricts the electronic space charge to thinner interfacial regions able to accumulate large amount of majority carriers, giving rise to the observed capacitance features. However, the issue of the commonly observed characteristic time scale ( $\sim$ s) remains unsolved. Such a slow time constant would indicate that mobile ions participate in the kinetics of electronic accumulation, although a comprehensive model integrating ion dynamics and electronic mechanisms still lacks. A few studies have pointed in this direction. The coupling between ion movement and surface polarization is specifically proposed, signaling that carriers show a retarded dynamics due to the slow relaxation of the accompanying ionic charge (Ravishankar et al., 2017). In fact, recent experiments probing switched photoluminescence in $\mathrm{MAPbI}_{3}$ with symmetrical electrodes explain the time dependence of the electrical current by ion-induced modification of the doping profile, as shown in Fig. 6 (Li et al., 2018). Here, the drift of vacancies modulate the local electron and hole concentration and consequently the steady-state electronic properties. Other proposals consider a 
rather exotic light-enhanced ionic conductivity by several orders of magnitude, affecting the overall electrical response of PSCs under illumination (Kim et al., 2018), while the capacitance is explained by stoichiometric (ionic) polarization effects (Gregori et al., 2016).
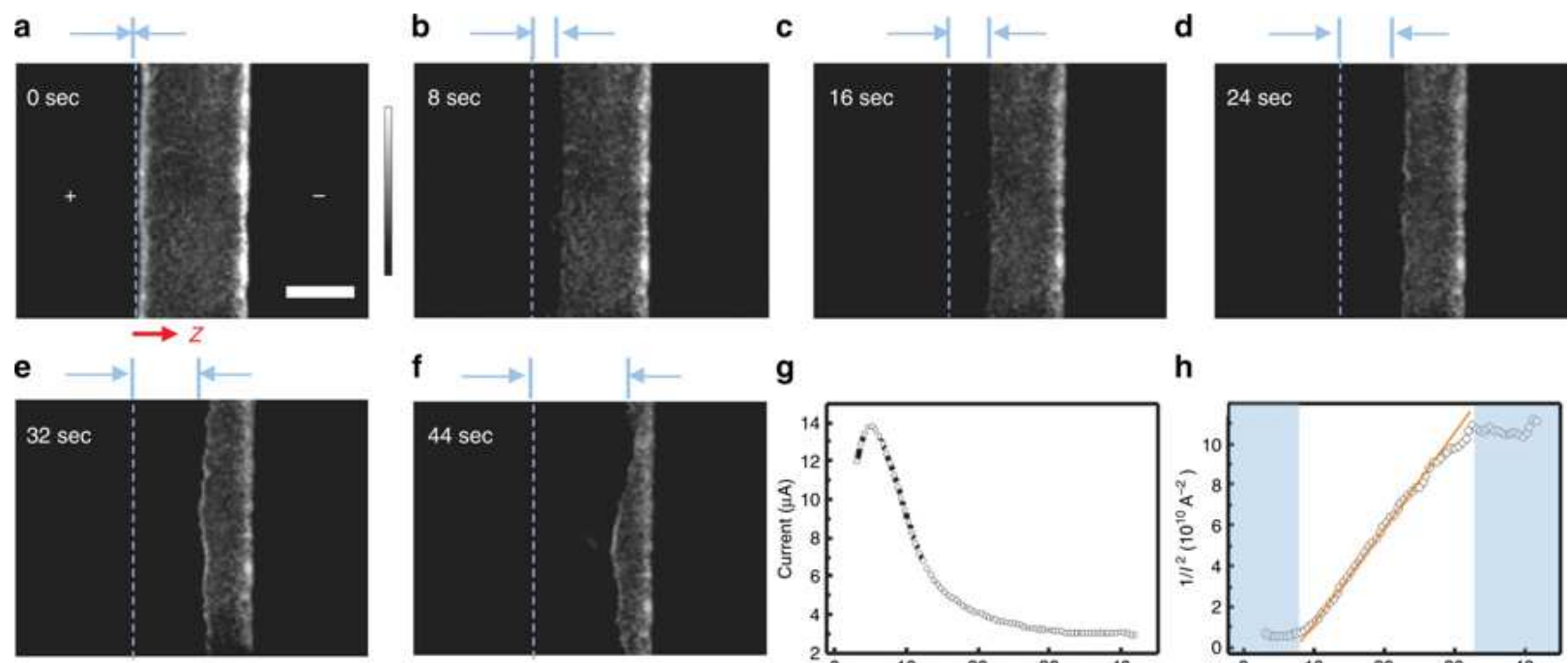

g

h
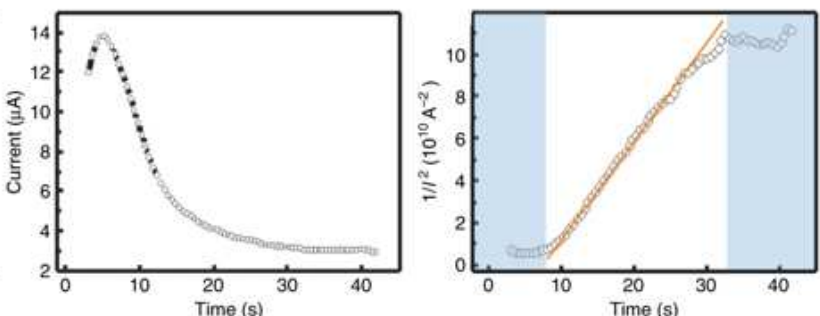

FIG. 6 Observation of a dark advancing front in a light-soaked perovskite. (a-f) Time-dependent PL images of a perovskite film $\mathrm{CH}_{3} \mathrm{NH}_{3} \mathrm{PbI}_{3-\mathrm{x}} \mathrm{Cl}_{\mathrm{x}}$ under an external electric field $\left(\sim 2 \times 10^{4} \mathrm{~V} \mathrm{~m}^{-1}\right)$. The "+ " and '-" signs indicate the polarity of the electrodes. The excitation intensity is $\sim 35 \mathrm{~mW} \mathrm{~cm}^{-1}$ with a wavelength of $440 \mathrm{~nm}$, and the exposure time per image is $200 \mathrm{~ms}$. The channel length is $\sim 150 \mu \mathrm{m} . z(t)$ represents the PL quenched areas. The scale bar represents $100 \mu \mathrm{m}$. The color bar is a gray value with arbitrary units, indicating the PL intensity. (g, h) Electrical current $I$ and $1 / I^{2}$, respectively, monitored as a function of time during the measurement of experiment (a). Reproduced under Creative Commons license (http://creativecommons.org/licenses/by/4.0/) from ref. (Li et al., 2018), Copyright (2018) Nature Publishing.

Previous works (Pockett et al., 2017) also suggested that the recombination mechanism in PSCs depends on the ionic environment, in such a way that ionic vacancies move to increase the recombination resistance on the ms to s timescale. It has been argued (Jacobs et al., 2018) that the coupling effect has an influence at low frequencies, as carrier recombination current becomes phase-delayed with respect to the voltage perturbation being responsible for observations of photoinduced capacitance. In this view, low-frequency capacitance is caused by out-of-phase 
carrier recombination instead of charge accumulation currents. Sophisticated equivalent circuits (ionically gated transistor interface) have been proposed (Moia et al., 2019) accounting for the ionic-electronic interplay governing charge injection and recombination. Similar arguments appear very recently but now disregarding ionic effects and highlighting delayed electronic injection currents as the sole cause of the low-frequency capacitive phenomena (Ebadi et al., 2019). These very recent studies show the still open character of this issue: whether large capacitive effects are explained exclusively from ionic or electronic mechanisms, or more likely an interplay between ionic kinetics and electronic charge accumulation/recombination is occurring (Jacobs et al., 2018; Moia et al., 2019; Ravishankar et al., 2017).

\section{Perovskite semiconductor regime}

The previous discussion leads us to consider if perovskites used as solar absorbing materials may be considered as relaxation semiconductors or not. Given the dielectric constant of perovskites $\varepsilon \approx 25-35$ (Almora et al., 2017b), and also the large carrier mobility values reported for both electrons and holes in the range of $\mu \approx 1-100 \mathrm{~cm}^{2} \mathrm{~V}^{-1} \mathrm{~s}^{-1}$ (Herz, 2017), small carrier density are enough in order to fulfill the relaxation semiconductor regime. Typical dielectric relaxation time values are plotted in Fig. 7 assuming $\sigma=q n \mu$ as a function of the carrier concentration $n$. As expected, long $\tau_{\mathrm{die}}$ is inferred as doping level approaches intrinsic values. In order to elucidate if $\tau_{\text {die }}>\tau_{\text {rec }}$ (relaxation semiconductor regime), an estimation of the recombination time is needed. Typical recombination times for perovskite materials can be calculated by recalling the long diffusion lengths $L_{\mu}$ reported in the literature exceeding $1 \mu \mathrm{m}$ (Stranks et al., 2013). From the known relation between recombination time and diffusion length $L_{\mu}=\sqrt{D_{\mu} \tau_{\text {rec }}}$ and assuming the Einstein expression for the diffusion coefficient $D_{\mu}=q k_{\mathrm{B}} T \mu$, 
one can readily calculate the carrier density for which $\tau_{\text {rec }}=\tau_{\text {die }}$ that results in $n \approx 4 \times 10^{13} \mathrm{~cm}^{-3}$, independently of the carrier mobility. This last carrier concentration entails that doping levels of order $<10^{13} \mathrm{~cm}^{-3}$ easily result in satisfying $\tau_{\text {die }}>\tau_{\text {rec }}$, a regime for which the chemical capacitance does not operate. This low value for the carrier density would signal the rather intrinsic (low doping) character of the perovskite compounds used as absorbing semiconductors in photovoltaics. This low value for the carrier density is in conflict with the doping levels usually encountered for solution-processed polycrystalline perovskite layers used in solar cells $\left(\sim 10^{16}-10^{17} \mathrm{~cm}^{-3}\right)$ (Shao et al., 2014). Thus, suggesting that the chemical capacitance in PSCs is not observed because of the low effective density-of-states of the conduction band, as aforementioned, even satisfying the recombination regime.

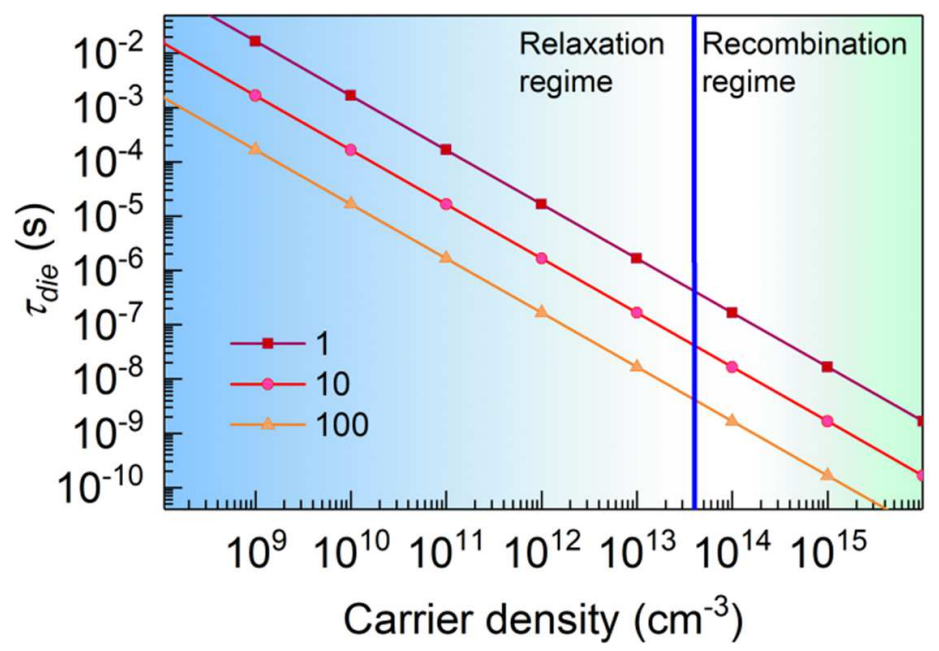

FIG. 7 Calculation of the dielectric relaxation time as a function of the carrier density for different mobility values in the range of $\mu \approx 1-100 \mathrm{~cm}^{2} \mathrm{~V}^{-1} \mathrm{~s}^{-1}$ for $\varepsilon \approx 30$, with an indication of the carrier density delimiting semiconductor regimes. 


\section{Concluding remarks}

In summary, contrary to that reported for silicon solar cells capacitances extracted in perovskite solar cells can hardly be interpreted in terms of the established formulation of chemical capacitances. Neither the low- nor high-frequency capacitive responses show the expected exponential trends and values. Here we propose two different explanations: $(i)$ the low effective density-of-states of the conduction band that implies chemical capacitances presumably masked by the geometrical capacitance values as $C_{\mu} \approx C_{g}$. (ii) The fact that perovskite compounds can be considered as relaxation semiconductors $\left(\tau_{\text {die }}>\tau_{\text {rec }}\right)$ with long times for carriers to slowly achieve steady-state conditions. For this last explanation to be satisfied, perovskites should be rather intrinsic or slightly doped semiconductors. (iii) It cannot be completely discarded that mobile ions influence the electronically originated capacitance of so as to situate its occurrence in the low-frequency part of the spectra.

\section{ORCID}

Osbel Almora: https://orcid.org/0000-0002-2523-0203

Germà Garcia-Belmonte: https://orcid.org/0000-0002-0172-6175

\section{Author contributions}

O.A. and G.G.B. jointly wrote the manuscript.

\section{Acknowledgments}

We acknowledge funding from MINECO of Spain under Projects MAT2016-76892-C31-R. O.A. acknowledges the financial support from the VDI/VD Innovation + Technik GmbH (Project-title: PV-ZUM) and the SAOT funded by the German Research Foundation (DFG) in the framework of the German excellence initiative.

\section{Notes}

The authors declare no competing financial interest. 


\section{References}

Almora, O., Aranda, C., Garcia-Belmonte, G., 2018a. Do capacitance measurements reveal lightinduced bulk dielectric changes in photovoltaic perovskites? J. Phys. Chem. C 122(25), 13450-13454. https://doi.org/10.1021/acs.jpcc.7b11703.

Almora, O., Aranda, C., Zarazua, I., Guerrero, A., Garcia-Belmonte, G., 2016. Noncapacitive hysteresis in perovskite solar cells at room temperature. ACS Energy Lett. 1(1), 209-215. https://doi.org/10.1021/acsenergylett.6b00116.

Almora, O., Cho, K.T., Aghazada, S., Zimmermann, I., Matt, G.J., Brabec, C.J., Nazeeruddin, M.K., Garcia-Belmonte, G., 2018b. Discerning recombination mechanisms and ideality factors through impedance analysis of high-efficiency perovskite solar cells. Nano Energy 48, 63-72. https://doi.org/10.1016/j.nanoen.2018.03.042.

Almora, O., García-Batlle, M., Garcia-Belmonte, G., 2019a. Utilization of temperature-sweeping capacitive techniques to evaluate band gap defect densities in photovoltaic perovskites. J. Phys. Chem. Lett. 10(13), 3661-3669. https://doi.org/10.1021/acs.jpclett.9b00601.

Almora, O., Gerling, L.G., Voz, C., Alcubilla, R., Puigdollers, J., Garcia-Belmonte, G., 2017a. Superior performance of $\mathrm{V}_{2} \mathrm{O}_{5}$ as hole selective contact over other transition metal oxides in silicon heterojunction solar cells. Sol. Energy Mater. Sol. Cells 168, 221-226. https://doi.org/10.1016/j.solmat.2017.04.042.

Almora, O., Lopez-Varo, P., Cho, K.T., Aghazada, S., Meng, W., Hou, Y., Echeverría-Arrondo, C., Zimmermann, I., Matt, G.J., Jiménez-Tejada, J.A., Brabec, C.J., Nazeeruddin, M.K., Garcia-Belmonte, G., 2019b. Ionic dipolar switching hinders charge collection in perovskite solar cells with normal and inverted hysteresis. Sol. Energy Mater. Sol. Cells 195, 291-298. https://doi.org/10.1016/j.solmat.2019.03.003.

Almora, O., Vaillant-Roca, L., Garcia-Belmonte, G., 2017b. Perovskite solar cells: A brief introduction and some remarks. Rev. Cubana Fis. 34(1), 58-68. http://www.revistacubanadefisica.org/index.php/rcf/article/view/RCF_34-1_58.

Almora, O., Zarazua, I., Mas-Marza, E., Mora-Sero, I., Bisquert, J., Garcia-Belmonte, G., 2015. Capacitive dark currents, hysteresis, and electrode polarization in lead halide perovskite solar cells. J. Phys. Chem. Lett. 6(9), 1645-1652. https://doi.org/10.1021/acs.jpclett.5b00480.

Azpiroz, J.M., Mosconi, E., Bisquert, J., De Angelis, F., 2015. Defects migration in methylammonium lead iodide and their role in perovskite solar cells operation. Energy Environ. Sci. 8(7), 2118-2127 https://doi.org/10.1039/C5EE01265A.

Bag, M., Renna, L.A., Adhikari, R.Y., Karak, S., Liu, F., Lahti, P.M., Russell, T.P., Tuominen, M.T., Venkataraman, D., 2015. Kinetics of ion transport in perovskite active layers and its implications for active layer stability. J. Am. Chem. Soc. 137(40), 13130-13137. https://doi.org/10.1021/jacs.5b08535.

Bergmann, V.W., Guo, Y., Tanaka, H., Hermes, I.M., Li, D., Klasen, A., Bretschneider, S.A., Nakamura, E., Berger, R., Weber, S.A.L., 2016. Local time-dependent charging in a perovskite solar cell. ACS Appl. Mater. Interfaces 8(30), 19402-19409. https://doi.org/10.1021/acsami.6b04104.

Bisquert, J., 2003. Chemical capacitance of nanostructured semiconductors: Its origin and significance for nanocomposite solar cells. Phys. Chem. Chem. Phys. 5(24), 5360-5364. https://doi.org/10.1039/B310907K. 
Bisquert, J., 2014. Nanostructured energy devices: Equilibrium concepts and kinetics. CRC Press Taylor \& Francis Group, Boca Raton.

Bisquert, J., Cahen, D., Hodes, G., Rühle, S., Zaban, A., 2004. Physical chemical principles of photovoltaic conversion with nanoparticulate, mesoporous dye-sensitized solar cells. J. Phys. Chem. B 108(24), 8106-8118. https://doi.org/10.1021/jp0359283.

Bisquert, J., Garcia-Belmonte, G., Mora-Sero, I., 2016. Characterization of capacitance, transport and recombination parameters in hybrid perovskite and organic solar cells, in: Da Como, E., De Angelis, F., Snaith, H.J., Walker, A. (Eds.), Unconventional thin film photovoltaics. The Royal Society of Chemistry, Cambridge, pp. 57-106. https://doi.org/10.1039/9781782624066-00057.

Bozano, L., Carter, A., Scott, J.C., Malliaras, G.G., Brock, P.J., 1999. Temperature- and fielddependent electron and hole mobilities in polymer light-emitting diodes. Appl. Phys. Lett. 74(8), 1132-1134. https://doi.org/10.1063/1.123959.

Brendel, R., 2005. Appendix b: Recombination, Thin-film crystalline silicon solar cells. WileyVCH Verlag GmbH \& Co. KGaA, pp. 209-239. https://doi.org/10.1002/3527603530.app2.

Burgelman, M., Grasso, C., 2004. Network of flatband solar cells as a model for solid-state nanostructured solar cells. J. Appl. Phys. 95(4), 2020-2024. https://doi.org/10.1063/1.1640787.

Carrillo, J., Guerrero, A., Rahimnejad, S., Almora, O., Zarazua, I., Mas-Marza, E., Bisquert, J., Garcia-Belmonte, G., 2016. Ionic reactivity at contacts and aging of methylammonium lead triiodide perovskite solar cells. Adv. Energy Mater. 6(9), 1502246 https://doi.org/10.1002/aenm.201502246.

Chen, B., Yang, M., Zheng, X., Wu, C., Li, W., Yan, Y., Bisquert, J., Garcia-Belmonte, G., Zhu, K., Priya, S., 2015. Impact of capacitive effect and ion migration on the hysteretic behavior of perovskite solar cells. J. Phys. Chem. Lett. 6(23), 4693-4700. https://doi.org/10.1021/acs.jpclett.5b02229.

Contreras-Bernal, L., Salado, M., Todinova, A., Calio, L., Ahmad, S., Idígoras, J., Anta, J.A., 2017. Origin and whereabouts of recombination in perovskite solar cells. J. Phys. Chem. C 121(18), 9705-9713. https://doi.org/10.1021/acs.jpcc.7b01206.

Ebadi, F., Taghavinia, N., Mohammadpour, R., Hagfeldt, A., Tress, W., 2019. Origin of apparent light-enhanced and negative capacitance in perovskite solar cells. Nat. Commun. 10(1), 1574. https://doi.org/10.1038/s41467-019-09079-z.

Fonash, S.J., 2010. Solar cell device physics, 2nd ed. Academic Press, Burlington MA.

Giorgi, G., Fujisawa, J.-I., Segawa, H., Yamashita, K., 2013. Small photocarrier effective masses featuring ambipolar transport in methylammonium lead iodide perovskite: A density functional analysis. J. Phys. Chem. Lett. 4(24), 4213-4216. https://doi.org/10.1021/jz4023865.

Green, M.A., Dunlop, E.D., Levi, D.H., Hohl-Ebinger, J., Yoshita, M., Ho-Baillie, A.W.Y., 2019. Solar cell efficiency tables (version 54). 27(7), 565-575. https://doi.org/10.1002/pip.3171.

Green, M.A., Ho-Baillie, A., Snaith, H.J., 2014. The emergence of perovskite solar cells. Nat. Photon. 8(7), 506-514. https://doi.org/10.1038/nphoton.2014.134.

Gregori, G., Yang, T.-Y., Sonocrate, A., Grätzel, M., Maier, J., 2016. Ionic conductivity of organic-inorganic perovskites: Relevance for long-time and low frequency behavior, in: Nam-GyuPark, Grätzel, M., Miyasaka, T. (Eds.), Organic-inorganic halide perovskites 
photovoltaics. From fundamentals to device architectures. Springer, Switzerland, pp. 107135. https://doi.org/10.1007/978-3-319-35114-8_5.

Herz, L.M., 2017. Charge-carrier mobilities in metal halide perovskites: Fundamental mechanisms and limits. ACS Energy Lett. 2(7), 1539-1548. https://doi.org/10.1021/acsenergylett.7b00276.

Jacobs, D.A., Shen, H., Pfeffer, F., Peng, J., White, T.P., Beck, F.J., Catchpole, K.R., 2018. The two faces of capacitance: New interpretations for electrical impedance measurements of perovskite solar cells and their relation to hysteresis. J. Appl. Phys. 124(22), 225702. https://doi.org/10.1063/1.5063259.

Jeon, N.J., Noh, J.H., Kim, Y.C., Yang, W.S., Ryu, S., Seok, S.I., 2014. Solvent engineering for high-performance inorganic-organic hybrid perovskite solar cells. Nat. Mater. 13(9), 897903. https://doi.org/10.1038/nmat4014.

Juarez-Perez, E.J., Sanchez, R.S., Badia, L., Garcia-Belmonte, G., Kang, Y.S., Mora-Sero, I., Bisquert, J., 2014. Photoinduced giant dielectric constant in lead halide perovskite solar cells. J. Phys. Chem. Lett. 5(13), 2390-2394. https://doi.org/10.1021/jz5011169.

Kim, G.Y., Senocrate, A., Yang, T.-Y., Gregori, G., Grätzel, M., Maier, J., 2018. Large tunable photoeffect on ion conduction in halide perovskites and implications for photodecomposition. Nat. Mater. 17(5), 445-449. https://doi.org/10.1038/s41563-0180038-0.

Kim, H.-S., Lee, C.-R., Im, J.-H., Lee, K.-B., Moehl, T., Marchioro, A., Moon, S.-J., HumphryBaker, R., Yum, J.-H., Moser, J.E., Grätzel, M., Park, N.-G., 2012. Lead iodide perovskite sensitized all-solid-state submicron thin film mesoscopic solar cell with efficiency exceeding 9\%. Sci. Rep. 2, 591. https://doi.org/10.1038/srep00591.

Kim, S., Bae, S., Lee, S.-W., Cho, K., Lee, K.D., Kim, H., Park, S., Kwon, G., Ahn, S.-W., Lee, H.-M., Kang, Y., Lee, H.-S., Kim, D., 2017. Relationship between ion migration and interfacial degradation of $\mathrm{ch}_{3} \mathrm{nh}_{3} \mathrm{pbi}_{3}$ perovskite solar cells under thermal conditions. Sci. Rep. 7(1), 1200. https://doi.org/10.1038/s41598-017-00866-6.

Lee, M.M., Teuscher, J., Miyasaka, T., Murakami, T.N., Snaith, H.J., 2012. Efficient hybrid solar cells based on meso-superstructured organometal halide perovskites. Science 338(6107), 643-647. https://doi.org/10.1126/science.1228604.

Li, C., Guerrero, A., Huettner, S., Bisquert, J., 2018. Unravelling the role of vacancies in lead halide perovskite through electrical switching of photoluminescence. Nat. Commun. 9(1), 5113. https://doi.org/10.1038/s41467-018-07571-6.

Li, C., Tscheuschner, S., Paulus, F., Hopkinson, P.E., Kießling, J., Köhler, A., Vaynzof, Y., Huettner, S., 2016. Iodine migration and its effect on hysteresis in perovskite solar cells. Adv. Mater. 28, 2446-2454. https://doi.org/10.1002/adma.201503832.

Lopez-Varo, P., Jiménez-Tejada, J.A., García-Rosell, M., Ravishankar, S., Garcia-Belmonte, G., Bisquert, J., Almora, O., 2018. Device physics of hybrid perovskite solar cells: Theory and experiment. Adv. Energy Mater. 8(14), 1702772. https://doi.org/10.1002/aenm.201702772.

Manser, J.S., Kamat, P.V., 2014. Band filling with free charge carriers in organometal halide perovskites. Nat. Photonics 8, 737-743. https://doi.org/10.1038/nphoton.2014.171.

Meloni, S., Moehl, T., Tress, W., Franckevičius, M., Saliba, M., Lee, Y.H., Gao, P., Nazeeruddin, M.K., Zakeeruddin, S.M., Rothlisberger, U., Graetzel, M., 2016. Ionic polarization-induced current-voltage hysteresis in $\mathrm{ch}_{3} \mathrm{nh}_{3} \mathrm{pbx}_{3}$ perovskite solar cells. Nat. Commun. 7, 10334. https://doi.org/10.1038/ncomms10334. 
Menéndez-Proupin, E., Palacios, P., Wahnón, P., Conesa, J.C., 2014. Self-consistent relativistic band structure of the $\mathrm{ch}_{3} \mathrm{nh}_{3} \mathrm{pbi}_{3}$ perovskite. Phys. Rev. B 90(4), 045207. https://doi.org/10.1103/PhysRevB.90.045207.

Moia, D., Gelmetti, I., Calado, P., Fisher, W., Stringer, M., Game, O., Hu, Y., Docampo, P., Lidzey, D., Palomares, E., Nelson, J., Barnes, P.R.F., 2019. Ionic-to-electronic current amplification in hybrid perovskite solar cells: Ionically gated transistor-interface circuit model explains hysteresis and impedance of mixed conducting devices. Energy Environm. Sci. https://doi.org/10.1039/C8EE02362J.

Mora-Sero, I., Garcia-Belmonte, G., Boix, P.P., Vazquez, M.A., Bisquert, J., 2009. Impedance spectroscopy characterisation of highly efficient silicon solar cells under different light illumination intensities. Energy Environ. Sci. 2(6), 678-686. https://doi.org/10.1039/B812468J.

Mora-Seró, I., Luo, Y., Garcia-Belmonte, G., Bisquert, J., Muñoz, D., Voz, C., Puigdollers, J., Alcubilla, R., 2008. Recombination rates in heterojunction silicon solar cells analyzed by impedance spectroscopy at forward bias and under illumination. Sol. Energy Mater. Sol. Cells 92(4), 505-509. https://doi.org/10.1016/j.solmat.2007.11.005.

Oga, H., Saeki, A., Ogomi, Y., Hayase, S., Seki, S., 2014. Improved understanding of the electronic and energetic landscapes of perovskite solar cells: High local charge carrier mobility, reduced recombination, and extremely shallow traps. J. Am. Chem. Soc. 136(39), 13818-13825. https://doi.org/10.1021/ja506936f.

Park, N.-G., 2015. Perovskite solar cells: An emerging photovoltaic technology. Materials Today 18(2), 65-72. https://doi.org/10.1016/j.mattod.2014.07.007.

Pockett, A., Eperon, G.E., Sakai, N., Snaith, H.J., Peter, L.M., Cameron, P.J., 2017. Microseconds, milliseconds and seconds: Deconvoluting the dynamic behaviour of planar perovskite solar cells. Phys. Chem. Chem. Phys. 19(8), 5959-5970. https://doi.org/10.1039/C6CP08424A.

Ravishankar, S., Almora, O., Echeverría-Arrondo, C., Ghahremanirad, E., Aranda, C., Guerrero, A., Fabregat-Santiago, F., Zaban, A., Garcia-Belmonte, G., Bisquert, J., 2017. Surface polarization model for the dynamic hysteresis of perovskite solar cells. J. Phys. Chem. Lett. 8(5), 915-921. https://doi.org/10.1021/acs.jpclett.7b00045.

Saliba, M., Matsui, T., Domanski, K., Seo, J.-Y., Ummadisingu, A., Zakeeruddin, S.M., CorreaBaena, J.-P., Tress, W.R., Abate, A., Hagfeldt, A., Grätzel, M., 2016. Incorporation of rubidium cations into perovskite solar cells improves photovoltaic performance. Science 354(6309), 206-209. https://doi.org/10.1126/science.aah5557.

Schiff, E.A., 2003. Low-mobility solar cells: A device physics primer with application to amorphous silicon. Sol. Energy Mater. Sol. Cells 78(1), 567-595. https://doi.org/10.1016/S0927-0248(02)00452-X.

Shao, Y., Xiao, Z., Bi, C., Yuan, Y., Huang, J., 2014. Origin and elimination of photocurrent hysteresis by fullerene passivation in $\mathrm{ch}_{3} \mathrm{nh}_{3} \mathrm{pbi}_{3}$ planar heterojunction solar cells. Nat. Commun. 5, 5784. https://doi.org/10.1038/ncomms6784.

Snaith, H.J., Abate, A., Ball, J.M., Eperon, G.E., Leijtens, T., Noel, N.K., Stranks, S.D., Wang, J.T.-W., Wojciechowski, K., Zhang, W., 2014. Anomalous hysteresis in perovskite solar cells. J. Phys. Chem. Lett. 5(9), 1511-1515. https://doi.org/10.1021/jz500113x.

Stranks, S.D., Eperon, G.E., Grancini, G., Menelaou, C., Alcocer, M.J.P., Leijtens, T., Herz, L.M., Petrozza, A., Snaith, H.J., 2013. Electron-hole diffusion lengths exceeding 1 
micrometer in an organometal trihalide perovskite absorber. Science 342(6156), 341-344. https://doi.org/10.1126/science.1243982.

Sze, S.M., Ng, K.K., 2007. Physics of semiconductor devices, 3rd ed. John Wiley \& Sons, Hoboken, New Jersey, USA.

Unger, E.L., Hoke, E.T., Bailie, C.D., Nguyen, W.H., Bowring, A.R., Heumuller, T., Christoforo, M.G., McGehee, M.D., 2014. Hysteresis and transient behavior in currentvoltage measurements of hybrid-perovskite absorber solar cells. Energy Environ. Sci. 7(11), 3690-3698. https://doi.org/10.1039/C4EE02465F.

van Roosbroeck, W., Casey, H.C., 1972. Transport in relaxation semiconductors. Phys. Rev. B 5(6), 2154-2175. https://doi.org/10.1103/PhysRevB.5.2154.

Wehrenfennig, C., Eperon, G.E., Johnston, M.B., Snaith, H.J., Herz, L.M., 2014. High charge carrier mobilities and lifetimes in organolead trihalide perovskites. Adv. Mater. 26, 15841589. https://doi.org/10.1002/adma.201305172.

Wei, J., Zhao, Y., Li, H., Li, G., Pan, J., Xu, D., Zhao, Q., Yu, D., 2014. Hysteresis analysis based on the ferroelectric effect in hybrid perovskite solar cells. J. Phys. Chem. Lett. 5(21), 3937-3945. https://doi.org/10.1021/jz502111u.

Yang, W.S., Noh, J.H., Jeon, N.J., Kim, Y.C., Ryu, S., Seo, J., Seok, S.I., 2015. Highperformance photovoltaic perovskite layers fabricated through intramolecular exchange. Science 348(6240), 1234-1237. https://doi.org/10.1126/science.aaa9272.

Zarazua, I., Bisquert, J., Garcia-Belmonte, G., 2016a. Light-induced space-charge accumulation zone as photovoltaic mechanism in perovskite solar cells. J. Phys. Chem. Lett. 7(3), 525528. https://doi.org/10.1021/acs.jpclett.5b02810.

Zarazua, I., Han, G., Boix, P.P., Mhaisalkar, S., Fabregat-Santiago, F., Mora-Seró, I., Bisquert, J., Garcia-Belmonte, G., 2016b. Surface recombination and collection efficiency in perovskite solar cells from impedance analysis. J. Phys. Chem. Lett. 7(24), 5105-5113. https://doi.org/10.1021/acs.jpclett.6b02193.

Zarazua, I., Sidhik, S., Lopéz-Luke, T., Esparza, D., De la Rosa, E., Reyes-Gomez, J., MoraSeró, I., Garcia-Belmonte, G., 2017. Operating mechanisms of mesoscopic perovskite solar cells through impedance spectroscopy and j-v modeling. J. Phys. Chem. Lett. 8, 60736079. https://doi.org/10.1021/acs.jpclett.7b02848.

Zhao, Y., Zhu, K., 2016. Organic-inorganic hybrid lead halide perovskites for optoelectronic and $\begin{array}{lllll}\text { electronic applications Chem. Soc. Rev. 45(3), 655-689. } & \text {. }\end{array}$ https://doi.org/10.1039/C4CS00458B 